\title{
Correction to: One-pot synthesis of highly stable and concentrated silver nanoparticles with enhanced catalytic activity [DOI:10.1007/s11814-019-0270-6]
}

\author{
Fiaz Hussain $^{* * *}$, Samy M. Shaban*,**, Jinhwan Kim ${ }^{*, \dagger}$, and Dong-Hwan Kim ${ }^{*, *, \dagger}$ \\ *School of Chemical Engineering, Sungkyunkwan University, Suwon 16419, Korea \\ **Biomedical Institute for Convergence at SKKU (BICS), Sungkyunkwan University, Suwon 16419, Korea \\ ***Polymer Department, Sungkyunkwan University, Suwon 16419, Korea \\ (Received 19 January 2019• Revised 11 February 2019 • Accepted 8 April 2019)
}

The typo in the acknowledged grant number needs to be corrected:

\section{ACKNOWLEDGEMENT}

This work was supported by the National Research Foundation of Korea (NRF) grant (R201901411).

R201901411 > R201901410

The authors apologize for this mistake. 